\author{
Universidade de São Paulo \\ Escola de Enfermagem De Ribeirão Preto \\ Programa de Pós Graduação em Enfermagem Fundamental
}

\title{
Sistematização da Assistência de Enfermagem: Proposta de um Software - Protótipo
}

Dircelene Jussara Sperandio

Dissertação de Mestrado apresentada ao Programa de Pós-Graduação em Enfermagem Fundamental da Escola de Enfermagem de Ribeirão Preto - USP.

Linha de pesquisa: Dinâmica de organização dos Serviços de Saúde e de Enfermagem.

Ribeirão Preto

2002 


\title{
Dircelene Jussara Sperandio
}

\section{Sistematização da Assistência de Enfermagem:}

\section{Proposta de um Software - Protótipo}

\author{
Dissertação de Mestrado apresentada ao \\ Programa de Pós-Graduação em \\ Enfermagem Fundamental da Escola de \\ Enfermagem de Ribeirão Preto - USP. \\ Linha de pesquisa: Dinâmica de \\ organização dos Serviços de Saúde e de \\ Enfermagem.
}

Orientadora:Profa. Dra. Yolanda Dora Martinez Évora

Ribeirão Preto 


\section{Sperandio, Dircelene Jussara}

Sistematização da Assistência de Enfermagem: Proposta de um software - Protótipo. Ribeirão Preto, 2002. 67 p. : il. ; $29,7 \mathrm{~cm}$.

Dissertação (Mestrado) - Escola de Enfermagem de Ribeirão Preto da Universidade de São Paulo/Departamento de Enfermagem Geral e Especializada.

Orientadora: Profa. Dra. Yolanda Dora Martinez Évora

1. Enfermagem. 2. Planejamento da Assistência. 3. Informática. 4. Software 
Data da Defesa: 19/12/2002

\section{BANCA EXAMINADORA}

Prof $^{a}$.Dr ${ }^{\mathrm{a}}$. Yolanda Dora Martinez Évora

Julgamento:

Assinatura

Prof.Dr. Marcio Mattos Borges de Oliveira

Julgamento:

Assinatura

Prof $^{\mathrm{a}}$.Dr ${ }^{\mathrm{a}}$. Ione Carvalho Pinto

Julgamento:

Assinatura 
Ao Pedro,

Meu esposo e

incentivador na busca

de vitóriase

apoio imprescindível

nos árduos momentos

E acima de tudo,

pelo colorido e

brilho conferidos

a minha existência.

Dovalantino e Ana,

Meus pais,

por me prepararem para a vida

com muito carinho e determinação.

E sobretudo, por investirem

e acreditarem em mim.

Evandro,

Meu irmão, pela eterna amizade e por constantemente

ensinar-me

a ver a vida so6

uma nova perspectiva. 


\section{AGRADECIMENTOS}

Meus agradecimentos especiais, ao meu Deus, que ilumina, protege e inspira constantemente minha trajetória nesta vida.

Agradeço também,

Á Profa. Dra. Yolanda Dora Martinez Évora, orientadora deste estudo, com quem compartilhei uma convivência harmoniosa e amiga e que contribuiu sobremaneira para

o meu aperfeiçoamento profissional e pessoal.

Á Profa. Dra. Dulce Maria Silva Vendrúsculo, coordenadora do Curso de Enfermagem da Faculdade de Enfermagem de Catanduva-FEC, pela disposição em mostra-me oinício deste caminho.

Á Dr. Hélder Célio Gandaio Ferreira (in memorian) cujo entusiasmo e estímulo impulsionaram a concretização deste estudo.

Á Alessandro Aparecido Sandrini e Marcelo Daltin Longhitano, da empresa Stars Computers, por acreditarem no meu trabalho e pela disponibilidade e atenção que possibilitaram o desenvolvimento do software.

Á Letícia, Mariane e Soraya, funcionárias e colegas da FEC, pelo carinho, eficiência e dedicação que proporcionam um convívio fraterno e prazeroso no nosso trabalho.

Á Cristiane, Juliano, Marisa, Solange e Zildinha, da Biblioteca FAMECA/FEC, pelo profissionalismo e presteza dispensados durante a elaboração desta dissertação.

Á Márcia, Maria Cristina, Maristela e Vanda, professoras e amigas da FEC, pela amizade, compreensão e solidariedade que sempre me receberam nas alegrias e dificuldades no percorrer desta trajetória.

Aos alunos de graduação da FEC, que possibilitam a oportunidade para eu ensinar e principalmente aprender.

Ao Corpo Docente da FEC, pelo estímulo, apoio e confiança nas atividades de ensino.

Ao Serviço de Enfermagem do Hospital Escola Emílio Carlos e Hospital Padre Alбino, pela satisfação e oportunidade de trabalharmos juntos.

Á Fundação Padre Albino, pelo apoio administrativo que facilitou a realização deste trabalho e proporcionou o meu crescimento profissional. 


\section{SUMÁRIO}

RESUMO

ABSTRACT

RESUMEN

LISTA DE FIGURAS

APRESENTAÇÃO

A mudança de paradigma na trajetória profissional-

1. INTRODUÇÃO

2. JUSTIFICATIVA DO ESTUDO E OBJETIVOS

3. SOFTWARE

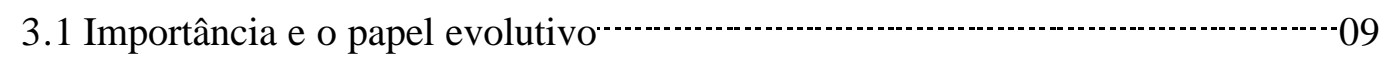

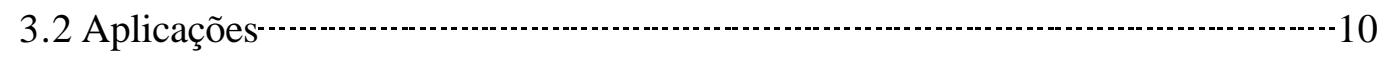

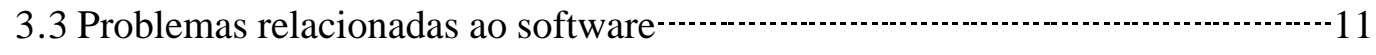

3.4 Desafios

4. A ENFERMAGEM NA ERA DIGITAL

5. INFORMÁTICA E A SISTEMATIZAÇÃO DA ASSISTÊNCIA DE

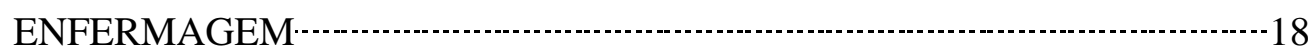

6. O PROCESSO DE ENFERMAGEM E AS INTERVENÇÕES DE ENFERMAGEM 
7. A ELABORAÇÃO E A IMPLANTAÇÃO DA SISTEMATIZAÇÃO DA ASSISTÊNCIA DE ENFERMAGEM NA UNIDADE DE TERAPIA SEMIINTENSIVA

8. O DESENVOLVIMENTO DO SOFTWARE PARA A SISTEMATIZAÇÃO

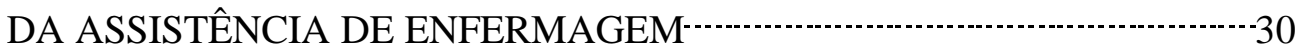

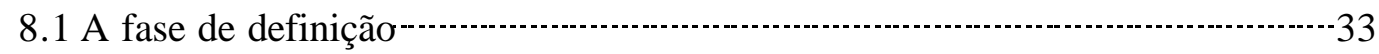

9.1.1 Planejamento

9.1.2 Análise e definição dos requisitos ……................................................-36

9.1.3 Revisão

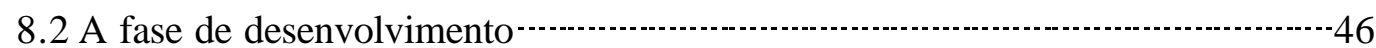

9. CONSIDERAÇÕES FINAIS

10. REFERÊNCIAS BIBLIOGRÁFICAS 


\section{RESUMO}

\section{SPERANDIO, D. J. Sistematização da Assistência de Enfermagem: Proposta de um}

software - protótipo . 2002. 67p. Dissertação Mestrado - Escola de Enfermagem de Ribeirão Preto, Universidade de São Paulo, Ribeirão Preto.

O propósito deste estudo foi desenvolver um software-protótipo, que possibilite aos enfermeiros atender ao planejamento da assistência de enfermagem, prescrição de intervenções de enfermagem e toda sua documentação de forma informatizada.A equipe multiprofissional envolvida no desenvolvimento deste software-protótipo foi constituída pela pesquisadora, um analista de sistema e um programador. A metodologia utilizada fundamentou-se no ciclo de vida de desenvolvimento de sistema, baseando-se no conceito de prototipação. Sedimentou-se em duas fases: a fase de definição e a de desenvolvimento. A fase de definição iniciou-se com a etapa de planejamento, seguido pela definição e análise dos requisitos necessários para sua construção e culminou com a produção da especificação de requisitos do software. A fase de desenvolvimento traduziu o conjunto de requisitos em um modelo informatizado, estruturado em 10 módulos, referentes ao processo de sistematização da assistência de enfermagem. Os módulos denominados: Ficha de Identificação, Dados Clínicos, Internações, Informações adicionais e Entrevista armazenam dados relativos às necessidades humanas básicas e abrangem: o índice de massa corpórea, situação clínica, resumo de admissão, internações anteriores e os dados para identificação do paciente. O módulo para Coleta de Dados foi desenhado para cadastrar informações diferenciadas sobre os sinais e sintomas e gerar, automaticamente, o módulo Lista de Problemas. Este viabiliza a elaboração da prescrição de enfermagem específica para cada paciente por meio da utilização de uma base de dados previamente estabelecida pelo sistema. Para propiciar maior comodidade e agilidade nas atividades de documentação, o módulo sobre Sinais Vitais permite transformar, eletronicamente, os valores atribuídos à pressão arterial, pulso, respiração e temperatura em gráficos individualizados. O ambiente Balanço Hidroeletrolítico permite implementar, automaticamente, o balanço parcial e total oferecendo aos enfermeiros simplicidade na execução desta tarefa, bem como realizar seu acompanhamento posterior. A avaliação deste recurso inovador na performance da Sistematização da Assistência de Enfermagem nos diferentes estágios do seu processo será objeto de um estudo posterior

Palavras-Chaves: Planejamento de assistência, Enfermagem, Informática, Software. 


\begin{abstract}
SPERANDIO, D. J. Patient Care System: Proposal of a software - prototype. 2002. 67p. Dissertação Mestrado - Escola de Enfermagem de Ribeirão Preto, Universidade de São Paulo, Ribeirão Preto.
\end{abstract}

The purpose of this study is to develop a software-prototype to help the nurses to plan the nursing care, to make nursing interventions and all documentation in a computerized way. The multi- professional team is involved in the development of this software-prototype and constituted by the researcher, a system analyst and a programmer. The methodology is based in the life cycle of system development, basing on the prototype concept. It is following up into two phases: the definition and the development one. The definition phase began with the planning stage, following for the definition and analysis of the requirements for the construction and it culminated with the specification of the software requirements. The development phase translated the group of requirements in a computerized model, structured in 10 modules, regarding the process of nursing care system. The Identification, Clinical Data, Interview and additional Information modules store data related to the basic human needs and they include: the index of corporal mass, clinical situation, admission summary, and the patient's identification. The Data Collection module was design to register information related to the signs and symptoms and to generate, automatically, the List of Problems module. This makes possible the elaboration of the nursing prescription of each patient using the data base established previously by the system. To make better the documentation activities, the Vital Signs module allows to transform, electronically, the values attributed to the blood pressure, pulse, breathing and temperature in individualized graphs. The fluid and electrolyte metabolism balance module allows to implement, automatically, the partial and total response helping the nurses in the execution of this task, as well as to accomplish the subsequent attendance. The evaluation of this innovative resource in the performance of Nursing Care System will be object of a subsequent study.

Key words: Patient Care Planning, Nursing, Informatics, Software. 


\section{RESUMEN}

\section{SPERANDIO, D. J. Sistema del Cuidado de enfermeria: Propuesta de un software - prototipo 2002. 67p. Dissertação Mestrado - Escola de Enfermagem de Ribeirão Preto, Universidade de São Paulo, Ribeirão Preto.}

El propósito de este estudio fue desarrollar un software-prototipo, que posibilite a los enfermeros atender al planeamiento do cuidado de enfermeria, prescripción de intervenciones de enfermeria y toda su documentación de forma informatizada. El equipo multiprofesional envolvida en el desarrollo de este software-prototipo fue constituída por la pesquisadora, un analista del sistema y un programador. La metodología utilizada se fundamentó en el ciclo de vida de desarrollo del sistema, basandose en el concepto de prototipo. Se sedimentó en dos fases: la definición y planeamiento. La fase de definición empezó con la fase de planeamiento, seguido por la definición y análisis de los requisitos necesarios para su construcción y culminó con la producción de la especificación de los requisitos del software. La fase de desarrollo tradujo el conjunto de requisitos en un modelo informatizado, estructurado en 10 módulos, referentes al proceso de sistematización del cuidado de enfermeria. Lo módulos denominados: Ficha de Identificación, Datos Clínicos, Internamiento, Informaciones adicionales y Entrevistas almacenan datos relativos a las necesidades humanas básicas y incluyen: el índice de masa corpórea, situación clínica, resumen de admisión, internamientos anteriores y los datos para identificación del paciente. El módulo para Colección de Datos fue dibujado para registrar informaciones diferenciadas sobre las señales y síntomas y generar, automáticamente, el módulo Lista de Problemas. Este hace posible la elaboración de la prescripción de enfermeria específica para cada paciente por medio de la utilización de una base de datos previamente establecida por el sistema. Para propiciar mayor comodidad y agilidad en las actividades de documentación, el módulo sobre Señales Vitales permite transformar, electrónicamente, los valores atribuidos à tensión arterial, pulso, respiración y temperatura en gráficos individualizados. El módulo de fluido y el electrólito equilibrio permite implementar, automáticamente, el resultado parcial y total ofreciendo a los enfermeros simplicidad en la ejecución de esta tarea, así como realizar su acompañamiento posterior. La evaluación de este recurso innovador en la actuación de desarrollar la sistematización del cuidado de enfermeria en los distintos estadios de su proceso será objeto de un estudio posterior.

Las palabras de la llave: Planificación de atención al paciente. Enfermeria, Informatica, Programas de computación. 
